\title{
La discriminación por edad: un estudio comparativo entre estudiantes universitarios
}

\author{
Margarita Gutiérrez; Teresa Mayordomo
}

How to cite this article:

Gutiérrez, M., \& Mayordomo, T. (2019). Age discrimination: a comparative study among university students. Acta Colombiana de Psicología, 22(2), 53-61. doi: http://www.doi.org/10.14718/ACP.2019.22.2.4

Recibido, julio 4/2018; Concepto de evaluación, octubre 24/2018; Aceptado, noviembre 13/2018

\author{
Margarita Gutiérrez* \\ Universidad Católica de Valencia, San Vicente Mártir, España \\ ORCID: https://orcid.org/0000-0001-5247-1777 \\ Teresa Mayordomo \\ Universidad Católica de Valencia, San Vicente Mártir, España \\ ORCID: https://orcid.org/0000-0003-1873-4910
}

Resumen

\begin{abstract}
Diversos estudios sobre estereotipos con estudiantes universitarios muestran que las personas mayores son percibidas más desde una imagen negativa que con estereotipos positivos. Teniendo en cuenta el creciente aumento de la población mayor en la actualidad, y con el fin de trabajar con personas mayores desde una visión positiva de sí mismos, es necesario que estos estereotipos desaparezcan. El objetivo de este trabajo fue determinar si existen estereotipos negativos hacia la vejez en estudiantes de carreras universitarias relacionadas y no relacionadas con el campo de la salud. En total, se evaluó a 262 estudiantes con el cuestionario CENVE, y, para saber si existían diferencias de estereotipos negativos entre los estudiantes según su titulación, se realizó un análisis ANOVA, además de comparaciones post hoc. Los resultados fueron significativos al nivel del $5 \%$ y evidenciaron la existencia de estereotipos negativos hacia la vejez en estudiantes de títulos universitarios relacionados y no relacionados con el campo de la salud. Los ANOVA mostraron diferencias para el CENVE total $\left(\mathrm{F}_{3,259}=\right.$ $\left.3.574 ; \mathrm{p}=.015 ; \eta^{2}=.040\right)$, para la dimensión de motivación social $\left(\mathrm{F}_{3,259}=3.697 ; \mathrm{p}=.012 ; \eta^{2}=.042\right)$ y para la dimensión de personalidad $\left(\mathrm{F}_{3,259}=5.157 ; \mathrm{p}=.002 ; \eta^{2}=.057\right)$. Las pruebas post hoc mostraron la existencia de diferencias en el grado de estereotipos negativos según las titulaciones en curso, donde las titulaciones sanitarias mostraron menor grado en comparación con las demás.

Palabras clave: discriminación por edad, personas mayores, estudiantes universitarios, estereotipos.
\end{abstract}

\section{Age discrimination: a comparative study among university students}

\begin{abstract}
Different studies with university students show the presence of a negative image about the elderly and an absence of positive stereotypes regarding this age group. With the increase of the older population, these stereotypes must disappear in order to work with aged people without a negative view of them. The aim of this paper was to identify the existence of negative stereotypes towards old age in students who are pursuing health-related and not related university degree courses. A total of 262 students were assessed with the CENVE questionnaire. In order to find out whether there were differences between students with respect to negative stereotypes, an ANOVA and a post-hoc comparison test were carried out. Results were significant at the 5\% level. These show evidence of the existence of negative stereotypes about old age in students pursuing university degrees related and not related to the field of health. ANOVAs showed differences in total CENVE $\left(\mathrm{F}_{3,259}=3.574\right.$; $\left.\mathrm{p}=0.015 ; \eta^{2}=0.040\right)$, Social Motivation dimension $\left(\mathrm{F}_{3.259}=3.697 ; \mathrm{p}=0.012 ; \eta^{2}=0.042\right)$, and Personality dimension $\left(\mathrm{F}_{3,259}=5.157 ; \mathrm{p}=0.002 ; \eta^{2}=0.057\right)$. The post-hoc tests show the existence of differences in stereotypes depending on the course that is being studied, where the health related courses show the lower level of negative stereotypes.

Key words: ageism, elderly, university students, stereotypes.
\end{abstract}

* San Vicente Mártir, c/ Sagrado Corazón, 5, Godella 46110, Valencia, España. Tel.: +34963637412, ext.5473 margarita.gutierrez@ucv.es 


\title{
A discriminaçáo por idade: um estudo comparativo entre estudantes universitários
}

\begin{abstract}
Resumo
Diversos estudos sobre estereótipos com estudantes universitários mostram que as pessoas idosas são percebidas mais frequentemente a partir de uma imagem negativa do que com estereótipos positivos. Levando em consideração o crescente aumento da população idosa na atualidade e como o fim de trabalhar com pessoas mais velhas a partir de uma visão positiva de si mesmos, é preciso que esses estereótipos desapareçam. O objetivo deste trabalho foi determinar se existem estereótipos negativos com respeito à velhice em estudantes de cursos universitários relacionados e não relacionados com o campo da saúde. No total, 262 estudantes foram avaliados com o questionário CENVE e, para saber se existiam diferenças de estereótipos negativos entre os estudantes de acordo com sua habilitação, foi realizada uma análise ANOVA, além de comparações post hoc. Os resultados foram significativos com um nível de 5\% e evidenciaram a existência de estereótipos negativos com respeito à velhice em estudantes de habilitações universitárias relacionadas e não relacionadas com o campo da saúde. Os ANOVA mostraram diferenças para o CENVE total $\left(\mathrm{F}_{3,259}=3.574 ; \mathrm{p}=.015 ; \eta^{2}=.040\right)$, para a dimensão de motivação social $\left(\mathrm{F}_{3,259}=3.697 ; \mathrm{p}=.012 ; \eta^{2}=.042\right)$ e para a dimensão de personalidade $\left(\mathrm{F}_{3,259}=5.157 ; \mathrm{p}=.002 ; \eta^{2}=.057\right)$. As provas post hoc mostraram a existência de diferenças no grau de estereótipos negativos de acordo com as habilitações em curso, sendo que as habilitações de saúde mostraram menor grau em comparação com as demais.

Palavras-chave: discriminação por idade, pessoas idosas, estudantes universitários, estereótipos.
\end{abstract}

\section{Introducción}

Con la mejora de la calidad de vida y la salud característica de estos tiempos, el envejecimiento de la población es una realidad demográfica en aumento. En España, por ejemplo, el $18.4 \%$ del total de la población en 2011 era mayor de 65 años, y se espera que llegue a $31.9 \%$ para el año 2049 (Abellán \& Esparza, 2011); y más recientemente, según la revisión de 2017 del informe Perspectivas de la Población Mundial (Naciones Unidas, 2017), se calcula que había aproximadamente 962 millones de personas con 60 años o más, es decir, un $13 \%$ de la población mundial, y se espera que el número de personas mayores se duplique para 2050 y se triplique para 2100 . Este evidente crecimiento de la población mayor está provocando una serie de cambios que tienen que ver tanto con las necesidades propias de las personas mayores como con el comportamiento y la actitud del resto de la población hacia ellas.

Al igual que con otros constructos sociales, la edad cronológica es una de las dimensiones por las cuales se categoriza de forma automática a los demás (Nelson, 2005), y una de estas categorizaciones -o estereotipos- tiene que ver con la idea que existe sobre las personas mayores con respecto al desarrollo de nuevas metas y objetivos, debido a que se considera que en esta etapa del ciclo vital estos intereses disminuyen. Sin embargo, como algunos autores han indicado, esto no es del todo cierto, ya que algunas de las metas vitales del ser humano se mantienen o, incluso, aumentan en la vejez (Mayordomo, Sales, Satorres \& Meléndez, 2016).

En este sentido, autores como Teixeira, Settembre y Leal (2007) señalan que un estereotipo se presenta cuando se ve a una persona mayor y se da por hecho que se conocen sus competencias, creencias y aptitudes. Específicamente, según Barón y Byrne (2005), los estereotipos son creencias referidas a características o rasgos típicos o modales que supuestamente poseen las personas pertenecientes a grupos sociales específicos, $y$, en particular, se acuñó el término "edadismo" (Butler, 1980) para referirse al proceso de elaboración de estereotipos y discriminación sistemática hacia las personas con edades mayores.

Al respecto, cabe señalar que algunos autores distinguen los estereotipos de otros fenómenos sociales con los que se encuentran relacionados -como las actitudes y los prejuicios-, pero Ayalon (2013) resalta la diferencia a partir de un componente conductual, que sería la discriminación; un componente afectivo, que hace referencia a los prejuicios; y un componente cognitivo de las actitudes, que sería lo que se conoce como estereotipos.

La investigación psicológica de los estereotipos negativos hacia la vejez (edadismo) es necesaria debido a que muchas de las creencias relacionadas con las personas mayores influyen negativamente en los hábitos de vida o auto-percepciones de los mayores, además de que pueden determinar los comportamientos de las demás personas en su relación con ellos, independientemente de su grupo etario, su grupo social, o su familiaridad con los mayores -incluidos los profesionales de la salud-(Bustillos, Fernández \& Ballesteros, 2013; Chalabaev et al., 2013; Fuentes, 2014; Kornadt \& Rothermund, 2015; León, Correa-Beltrán \& Giacaman, 2015; Rivera \& Paredez, 2014).

Como señalan Gázquez et al. (2009), estos estereotipos se nutren de ideas que no se corresponden con la realidad ni con los hallazgos científicos sobre la vejez y el envejecimiento; y, según Losada-Baltar (2004), tienen un importante impacto en el modo en que la sociedad en general y los 
cuidadores o profesionales de la salud en particular tratan a las personas mayores, lo cual puede propiciar conductas que perjudican su bienestar. Estas conductas o comportamientos que influyen en el mantenimiento de los estereotipos y su resistencia al cambio, por lo que, de acuerdo con la profecía autocumplida, las personas mayores pueden actuar ajustándose a la imagen negativa que perciben que se tiene de ellas (Levy, 2003); imagen que influye negativamente en el tipo de envejecimiento de los mayores y da lugar a un menor envejecimiento activo (Fernandez-Ballesteros, Olmos, Santacreu, Bustillos \& Molina, 2017).

La investigación en esta área tanto en el campo general como en la literatura profesional médica específica y de textos de gerontología (Robinson, Briggs \& O’Neill, 2012) ha demostrado que existen ideas falsas sobre el envejecimiento (Chrisler, Barney \& Palatino, 2017). En elárea profesional, por ejemplo, se ha encontrado que los sesgos edadistas favorecen una descripción de las personas mayores basada fundamentalmente en rasgos negativos que puede fomentar la realización de prácticas profesionales discriminatorias (Losada, 2004).

Según Casado, Bustillos, Vaquero, Casal y FernándezBallesteros (2016), la discriminación directa e indirecta se establece en algunos casos mediante el procedimiento de excluir o poner límites de edad difíciles de justificar a la hora de llevar a cabo medidas para la detección temprana de ciertas enfermedades, de modo que cuando se realizan diagnósticos, las personas mayores reciben con mayor frecuencia un diagnóstico de tipo orgánico-como la demencia- $y$ tienden a recibir tratamientos distintos - preferencia por tratamientos farmacológicos, por ejemplo-a los que se proporcionaría si la persona fuese más joven; lo cual, en algunos casos, podría favorecer el establecimiento de estilos de envejecimiento patológicos (Kydd \& Fleming, 2015).

Por otra parte, en lo que respecta a la salud mental, el mantenimiento de estereotipos negativos de edad contribuye en gran medida a la limitada atención que se proporciona a las personas mayores con problemas psicológicos; $y$, en este sentido, las creencias pesimistas de los propios profesionales -relacionadas con los cambios de personalidad y la tendencia a la rigidez, la demencia, la pérdida de funciones cognitivas y de capacidades en general, la tendencia al estado de ánimo depresivo y la soledad, entre otras- sobre las posibilidades de mejora a personas de esta edad contribuyen a cierto $\mathrm{ni}$ hilismo terapéutico que supone una barrera para el acceso a los servicios de intervención psicosocial por parte de esta población (Montorio, Márquez, Losada \& Fernández, 2003).

En España, incluso, algunas de estas formas de discriminación por la edad parecen estar socialmente aceptadas en el ámbito de la atención sanitaria a los mayores, ya que en en el Informe del Defensor del Pueblo del año 2000 se señala la falta de tratamientos de rehabilitación en logopedia, fisioterapia y terapia ocupacional para la población mayor.

Teniendo esto en cuenta, el papel de los profesionales de la salud es fundamental para derribar los falsos mitos sobre esta etapa de la vida, y es por ello que autores como Abreu y Caldevilla (2015) consideran que las actitudes de los alumnos universitarios del área sanitaria en la actualidad podrían influir en la calidad de la atención a la persona mayor en un futuro. En este sentido, existen estudios con alumnos universitarios en distintas áreas de conocimiento que muestran la presencia de una imagen negativa de la vejez y una ausencia de estereotipos positivos hacia las personas mayores, como es el caso de Campos y Salgado (2013), Chamorro (2014), Sandoval, Galindo, Figueroa, Pulido y Ruvalcaba (2016), y Sanhueza (2014).

Ahora bien, tomando en consideración el envejecimiento poblacional, es posible prever una mayor demanda de salud por parte de los adultos mayores, lo que implicaría también un gran número de profesionales del ámbito educativo y sociosanitario que trabajarán con población mayor, y que, de estar influidos por la imagen social negativa creada sobre la vejez, pueden tener una afectación perjudicial en sus actitudes y comportamientos a la hora de trabajar con dicha población. Respecto a esto, en los trabajos de Levy (2003) se demostró que en médicos y psiquiatras que trabajaban con personas mayores había una tendencia a atribuir despectivamente varios problemas a la vejez-como depresión, insomnio, falta de motivación, pérdida de capacidades-en lugar de intentar tratarlos como lo harían con una persona más joven; lo cual supone un desafío para las instituciones de educación superior, especialmente para las que ofrecen programas en el área de las ciencias de la salud, tal como habían señalado Mehrotra, Townsend y Berkman (2009).

En síntesis, los estereotipos son una imagen estructurada y aceptada por la mayoría de las personas, y, cuando ocurren hacia la vejez y los mayores, estas ideas suelen estar asociadas con aspectos negativos y patológicos como los relacionados con que los mayores son frágiles, intransigentes o dependientes (Bousfield \& Hutchison, 2010). Este tipo de actitudes, concepciones o percepciones que los estudiantes (futuros profesionales) tienen sobre las personas mayores puede jugar un papel importante en la forma en que posteriormente trabajarán con las personas de este grupo de edad (Zambrini, Moraru, Hanna, Kalache \& Nuñez, 2008). De esto surge la importancia del objetivo de este trabajo: evaluar la presencia de estereotipos negativos hacia la vejez en estudiantes universitarios de diferentes especialidades de formación universitaria, y conocer si hay diferencias en el nivel de estereotipos en función de la titulación universitaria que se está estudiando. 


\section{Método}

Diseño

Este estudio contó con un diseño de grupos naturales de carácter transversal, con muestreo no aleatorizado.

\section{Participantes}

En total, participaron 262 sujetos con media de edad de 21.29 años $(\mathrm{DT}=3.47), 18.6 \%$ hombres y $81.4 \%$ mujeres, todos alumnos de titulaciones universitarias privadas de primer a cuarto curso de su carrera universitaria. En cuanto al tipo de estudio que realizan, el $43.3 \%$ lo hacía en Psicología, el 19\%, en Educación, el 15.6 \% eran profesiones con posible contacto con mayores (Educación social, Pedagogía y Ciencias de la actividad física y deporte), y el $22.1 \%$ cursaban en el momento de la investigación titulaciones sanitarias que sí trabajarán con mayores en un futuro (Fisioterapia, Enfermería, Podología, Odontología y Terapia ocupacional). Como criterios de inclusión se solicitaba que estuvieran cursando estudios universitarios de grado y que dieran su consentimiento para participar en el estudio. Los alumnos que fueron informados y no firmaron su consentimiento para participar fueron excluidos del estudio.

\section{Instrumentos}

Todos los participantes respondieron a preguntas sociodemográficas relacionadas con su sexo, edad y la carrera que están estudiado, además del Cuestionario de Estereotipos Negativos hacia la Vejez (CENVE; Blanca, Sánchez \& Trianes, 2005), una escala compuesta por 15 ítems con un contenido negativo y estereotipado sobre el envejecimiento y los mayores.

Las autoras del CENVE recomiendan calcular tres subescalas sobre estereotipos negativos relacionados con (a) la salud, (b) el ámbito motivacional social y (c) el carácter y la personalidad, evaluadas a través de un análisis factorial. Cada dimensión está compuesta por cinco ítems -que se evalúan con una escala Likert de 1 a 4, según el nivel de acuerdo- con puntuaciones que pueden oscilar entre $5 \mathrm{y}$ 20 , en donde la puntuación que se encuentre entre 12.5 y 20 da cuenta de un alto grado de estereotipo negativo hacia la vejez. Esta prueba ha sido utilizada en otras investigaciones de forma completa - con la puntuación total- o por medio de las subescalas - por separado- (León et al., 2015; Sarabia \& Castanedo, 2015). Los coeficientes alfa de Cronbach obtenidos en el presente trabajo son satisfactorios tanto para el CENVE Total $(\alpha=.895)$ como para la dimensión de motivación social $(\alpha=.774)$, la dimensión de personalidad ( $\alpha=.758) \mathrm{y}$, en menor medida, la dimensión de $\operatorname{salud}(\alpha=.668)$.

\section{Procedimiento}

Para la recogida de la muestra se informó a los estudiantes sobre el estudio y se les contó el objetivo de la investigación y la forma de proceder si querían participar. Todos los que quisieron participar de forma voluntaria firmaron su consentimiento para, posteriormente, responder al cuestionario de forma completamente anónima y colectiva. Los participantes respondieron al cuestionario en el aula donde se imparte la docencia, en un tiempo aproximado de 20 minutos.

\section{Análisis}

Se llevaron a cabo análisis descriptivos (tendencia central y dispersión) y comparativos de las puntuaciones obtenidas en estereotipos negativos con el programa IBM SPSS, v. 23. En particular, se llevó a cabo un ANOVA (Rubio \& Berlanga, 2012) para identificar las diferencias en estereotipos negativos hacia la vejez en función de la titulación en curso. Los resultados se consideraron significativos al nivel de $5 \%$. Por último, se realizaron pruebas post hoc (Tukey) para dar cuenta de las titulaciones que daban las diferencias.

\section{Resultados}

Antes de indicar las diferencias encontradas tanto en la puntuación total de la escala como en las subescalas en función de la titulación en curso, es importante señalar que todas las carreras presentan estereotipos negativos hacia la vejez en mayor o menor medida.

Una vez analizadas las pruebas de normalidad y de homogeneidad de varianza medidas por medio del test de Kolmogorov-Smirnov -normalidad para el CENVE total $(\mathrm{K}-\mathrm{S}=.051 ; \mathrm{p}=.092)$, motivación social $(\mathrm{K}-\mathrm{S}=.053$; $\mathrm{p}=.089)$, salud $(\mathrm{K}-\mathrm{S}=.043 ; \mathrm{p}=.085)$ y personalidad $(\mathrm{K}-\mathrm{S}=.053 ; \mathrm{p}=.091)$ - $\mathrm{y}$ la prueba de Levene-homogeneidad de varianzas del CENVE total $\left(\right.$ Levene $_{3,258}=.477 ; \mathrm{p}=.698$ ), motivación social (Levene $\left._{3,258}=.124 ; \mathrm{p}=.946\right)$, salud (Levene $_{3,258}=.160 ; \mathrm{p}=.923$ ) y personalidad (Levene ${ }_{3,258}=$ $.561 \mathrm{p}=.642)-$, los ANOVA simples mostraron diferencias significativas entre las distintas titulaciones tanto para el CENVE total $\left(\mathrm{F}_{3,259}=3.574 ; \mathrm{p}=.015 ; \eta^{2}=.040\right)$ como para la motivación social $\left(\mathrm{F}_{3,259}=3.697 ; \mathrm{p}=.012 ; \eta^{2}=.042\right) \mathrm{y}$ la personalidad $\left(\mathrm{F}_{3,259}=5.157 ; \mathrm{p}=.002 ; \eta^{2}=.057\right)$, pero no para la dimensión de salud $\left(\mathrm{F}_{3,259}=1.194 ; \mathrm{p}=.313\right.$; $\left.\eta^{2}=.014\right)$. 
$\mathrm{Al}$ realizar las pruebas post hoc para el CENVE total se obtuvieron diferencias estadísticamente significativas entre el grupo de titulaciones sanitarias y aquellos que estudian Psicología $(\mathrm{p}=.026)$ y Educación $(\mathrm{p}=.001)$. En la Tabla 1 se pueden observar las medias obtenidas para cada una de estas titulaciones, siendo el grupo de titulaciones sanitarias el que menos estereotipos hacia la vejez muestra.

Tabla 1.

Descriptivos para el CENVE total

\begin{tabular}{lcc}
\hline \multicolumn{1}{c}{ Titulación } & Media & Desviación típica \\
\hline Psicología & 37.66 & 7.83 \\
Educación & 39.84 & 7.00 \\
Sanitarias & 34.03 & 8.64 \\
Con posible relación & 36.90 & 8.51 \\
\hline
\end{tabular}

Específicamente, con respecto a las pruebas post hoc para la dimensión de motivación social, se encontraron diferencias significativas entre las titulaciones sanitarias con Psicología $(\mathrm{p}=.004)$ y Educación $(\mathrm{p}=.002)$. En esta dimensión, el grupo de sanitarias no muestra estereotipo negativo hacia la vejez, mientras que el grupo de Educación y Psicología son los que muestran mayor estereotipo. En la Tabla 2 se pueden observar las medias obtenidas por los grupos.

Tabla 2.

Descriptivos para la dimensión de motivación social

\begin{tabular}{lcc}
\hline \multicolumn{1}{c}{ Titulación } & Media & Desviación típica \\
\hline Psicología & 12.95 & 2.98 \\
Educación & 13.42 & 2.77 \\
Sanitarias & 11.27 & 3.25 \\
Con posible relación & 12.29 & 3.31 \\
\hline
\end{tabular}

En lo que respecta a la dimensión de personalidad, se encontraron diferencias entre el grupo de titulaciones sanitarias y el resto de los grupos -Psicología $(p=.001)$, Educación $(\mathrm{p}=.001)$, y titulaciones con posible relación $(\mathrm{p}=.050)-$, siendo el grupo de sanitarias el que no muestra estereotipo negativo, mientras que el resto de grupos sí. En la Tabla 3 se pueden observar las puntuaciones obtenidas para los grupos.

Tabla 3.

Descriptivos para la dimensión de personalidad

\begin{tabular}{lcc}
\hline \multicolumn{1}{c}{ Titulación } & Media & Desviación típica \\
\hline Psicología & 12.81 & 2.92 \\
Educación & 13.84 & 2.73 \\
Sanitarias & 10.84 & 3.25 \\
Con posible relación & 12.43 & 3.25 \\
\hline
\end{tabular}

Finalmente, a pesar de que no existen diferencias estadísticamente significativas en la dimensión salud, es relevante presentar las medias obtenidas para todos los grupos establecidos (véase Tabla 4).
Tabla 4.

Descriptivos para la dimensión de salud

\begin{tabular}{lcc}
\hline \multicolumn{1}{c}{ Titulación } & Media & Desviación típica \\
\hline Psicología & 11.89 & 2.84 \\
Educación & 12.58 & 2.58 \\
Sanitarias & 11.91 & 2.87 \\
\hline
\end{tabular}

\section{Discusión}

El presente estudio muestra evidencia de la existencia de estereotipos negativos hacia la vejez en alumnos de titulaciones universitarias relacionadas y no relacionadas con el ámbito de la salud, además de la existencia de diferencias en estereotipos en función de la titulación que se está cursando.

Las diferencias encontradas en los estereotipos fueron estadísticamente significativas, al igual que en los resultados hallados en estudios similares (Chamorro, 2014). Concretamente, las diferencias se encuentran entre el grupo de titulaciones sanitarias, es decir, titulaciones como Psicología y Educación. En particular, los estudiantes estas titulaciones fueron el único grupo con bajos estereotipos negativos hacia las personas mayores, dato que en cierto modo sorprende, ya que se trata de estudiantes de profesiones vinculadas al tratamiento y la rehabilitación en procesos de envejecimiento que pueden considerarse patológicos, y el contacto con el conocimiento de la parte negativa de la vejez - asociada a la enfermedad y al deterioro- puede llevar a pensar que los alumnos de dichas titulaciones podrían tener una imagen más negativa de las personas mayores.

Esta idea de que el contacto con personas mayores dependientes reafirma el estereotipo social de enfermedad y deterioro asociado a la edad (Aristizábal-Vallejo, Morales, Salas \& Torres, 2009) se puede observar en varios estudios, como el trabajo de Sandoval et al. (2016), en donde se encuentran más estereotipos negativos en los estudiantes de carreras que tienen contacto directo con las personas de edad; al igual que en carreras relacionadas con la salud, como en Enfermería (Duran-Badillo et al., 2016; Sarabia \& Castanedo, 2015) o en Odontología (Fernández, Monardes, Díaz, Fuentes \& Padilla, 2017). Sin embargo, la ausencia de estereotipos negativos en el grupo de estudiantes de titulaciones de la salud del presente estudio puede tener que ver con la falta de contacto preprofesional y de intervención con personas mayores con patología, ya que se trata de alumnos en los primeros cursos de formación.

A pesar de que no se dé cuenta de estereotipos negativos en este grupo de estudiantes, es importante seguir trabajando en esta línea, ya que debido al cambio demográfico se espera un mayor número de adultos mayores que influirán 
en la demanda de salud, lo que implica que aquellos que ingresan en carreras del área de la salud, en gran media, trabajarán con personas mayores.

El siguiente grupo de alumnos al que se hace referencia y que sí muestra estereotipos negativos es el de los alumnos de la carrera de Educación. Se podría entender que el interés de estos estudiantes está puesto en las edades tempranas de la vida, y no en el envejecimiento; sin embargo, es importante tener en cuenta las creencias de los maestros, ya que estos son el principal agente socializador en la escuela, y hay aspectos sobre las ideas que tienen de la sociedad, la vida y las personas, que van a tener influencia en la educación de sus alumnos.

Siguiendo el orden de resultados, los estudiantes de Psicología, titulación que no se ha incluido dentro de las sanitarias directamente, muestran más estereotipos negativos que los alumnos de titulaciones sanitarias. Al respecto, hay que tener en cuenta que en la profesión del psicólogo se puede trabajar en el ámbito de la vejez desde las diferentes perspectivas del envejecimiento (saludable, activo, positivo, patológico, entre otros), y, como señala Losada-Baltar (2004), la discriminación hacia las personas mayores -en sus tres componentes: cognitivo, emocional y conductual-y los estereotipos negativos hacia esta población favorecen la descripción negativa de dichas personas, así como la realización de prácticas profesionales discriminatorias por este sesgo.

En referencia al área cognitiva, en donde el psicólogo puede ejercer una gran labor, distintas investigaciones señalan que los estudiantes universitarios presentan una dificultad especial para discriminar entre "mitos" y "realidades" respecto a los problemas cognitivos que pueden presentarse a medida que se envejece, por lo que existe una tendencia a homogeneizar a las personas mayores en torno a una idea de deterioro cognitivo patológico, inevitable y universal. Esta prioridad hacia los aspectos cognitivos del envejecimiento deja en un papel secundario la existencia de patologías psicológicas de índole emocional que se dan en cualquier etapa del ciclo vital, lo cual puede verse reflejado en la falta de estudios sobre la eficacia de psicoterapias en personas mayores (Márquez-González, 2010).

Con respecto a las subescalas del CENVE, en el presente trabajo se encontraron diferencias significativas entre el grupo de estudiantes de titulaciones sanitarias y el resto de las titulaciones, en particular, en dos de ellas: motivación social y personalidad.

Por lo que respecta a la subescala de salud, definida como la aparición de discapacidad, deterioro cognitivo y la existencia generalizada de enfermedades mentales en la vejez, no se encontraron diferencias significativas entre los grupos. No obstante, si se tienen en cuenta las puntuaciones obtenidas, el grupo de sanitarias es el que menos estereotipo presenta, lo que lleva a pensar que tener una formación en el área, aunque sea desde la perspectiva patológica, ofrece una visión más amplia sobre la variabilidad del envejecimiento que protege frente a los prejuicios que se observan en estudiantes de otras titulaciones.

Por otra parte, en la dimensión de motivación social -relacionada con las carencias afectivas, falta de intereses vitales y capacidad disminuida para desempeñar una actividad laboral-se observan diferencias entre las titulaciones sanitarias -cuyas puntuaciones muestran bajo estereotipo negativo hacia la vejez- y el resto de las titulaciones, a excepción de las titulaciones que pueden tener un posible contacto con personas mayores. Concretamente, los estudiantes de Educación son los que más estereotipos negativos presentan, aspecto que debe ser tenido en cuenta dentro de su formación, dado que en sus practica profesional serán trasmisores de valores y creencias a sus estudiantes. Este alumnado (los futuros maestros) tiene la idea de que los mayores tienen problemas a la hora de socializarse, aspecto llamativo en la escuela de hoy en día, donde los abuelos tienen cada vez más un papel importante y participativo en los centros educativos, siendo agentes socializadores importantes para sus nietos (Sanz, Mula \& Moril, 2011).

Resulta llamativo que después de los futuros maestros sean los estudiantes de Psicología quienes tengan más creencias relacionadas con la incapacidad del mayor para resolver problemas, así como con una pérdida de interés por las cosas y una disminución de sus amistades, ya que dentro del plan de estudios de Psicología se trabaja de forma significativa la capacidad humana de adaptación a los cambios; y aunque en esta etapa muchas situaciones a las que se enfrentan tienen un elemento de pérdida y se encuentran en un contexto sobre el cual no se tiene control personal, algunos autores, como Carver y Connor-Smith (2010) indican que a medida que se envejece las personas adquieren más habilidad para adaptarse por medio de estrategias de afrontamiento centradas en la emoción, y que al envejecer los individuos tienden a actuar ante una situación estresante tomando distancia, piensan más las cosas, conocen mejor sus emociones y elaboran mejores y más apropiadas estrategias para actuar eficazmente ante situaciones conflictivas.

Incluso, dentro de las asignaturas de grado de Psicología (a través del desarrollo psicosocial y otras materias), también se ve cómo se producen cambios a nivel social en la vejez, y aunque es cierto que hay un descenso de este tipo de actividad (Charles \& Carstensen, 2009), también existe la tendencia a seleccionar los contactos sociales ya en la mediana edad, lo que hace que este descenso sea muy selectivo y que afecte sobre todo a los contactos más 
superficiales, mientras que las relaciones más estrechas y proveedoras de apoyo permanecen básicamente intactas con la edad (Meléndez, Tomás \& Navarro, 2007).

Por último, en la dimensión de personalidad, que hace alusión a los problemas de labilidad emocional y cambios en la personalidad -como la rigidez-, los resultados del presente estudio sugieren que los estudiantes de carreras sanitarias muestran menos estereotipos negativos, lo cual puede tener una influencia en su forma de intervención, diagnóstico y actitud en su desempeño laboral con las personas mayores en el futuro. Sin embargo, se encontraron estereotipos negativos en esta dimensión en los alumnos pertenecientes a las otras titulaciones, siendo el grupo de alumnos de Educación el que mayores puntuaciones mostró, seguido por el grupo de titulaciones con posible relación (como los estudiantes de Educación social y Ciencias de la actividad física), y, finalmente, por los estudiantes de Psicología. Nuevamente, es importante señalar que el grupo de Psicología muestre estereotipos en dicha subescala, dado que esta profesión tiene como estudio el desarrollo de la personalidad. Sin embargo, este nivel de formación en el área de la personalidad puede ser tan específico para el estudiante de Psicología que, si no se ofrece un contenido específico de la idiosincrasia del envejecimiento, puede dar cabida a prejuicios por parte de los alumnos que se centran en las características de personalidad.

En conclusión, y como se ha mencionado, un mayor nivel de formación sobre la vejez y el envejecimiento disminuye la actitud negativa hacia la vejez (Sarabia \& Castanedo, 2015), lo que hace necesario, como ya señalaban Sandoval et al. (2016), revisar los programas de formación de las actuales titulaciones universitarias para introducir aspectos relacionados con el envejecimiento, la vejez y las personas mayores de manera más realista y sin prejuicios, en donde se tenga en cuenta que el envejecimiento se relaciona con todos los campos de la vida humana y social, y no solo con la salud. Finalmente, la formación y el posterior desarrollo profesional de los estudiantes puede influir en la relación y atención con las personas mayores, y la presencia de estereotipos negativos en los estudiantes del presente estudio puede ofrecer pistas sobre hacia dónde dirigir la intervención para frenar la discriminación por edad.

Como limitaciones del estudio se puede señalar el tamaño de la muestra y las titulaciones analizadas, ya que podría ser interesante añadir en próximas investigaciones más titulaciones relacionadas y no relacionadas con la vejez y con la salud, y buscar una cantidad similar de participantes por grupo. Asimismo, es importante resaltar la baja magnitud del efecto encontrada, lo que indica que la titulación es solo una variable que diferencia pero no predice los estereotipos negativos hacia los adultos mayores. Una posible línea de investigación a futuro sería el desarrollo de programas de intervención para alumnos de Psicología, ya que la proyección demográfica y social hacen necesario un perfil de psicólogo con una visión realista y actual de la vejez; aspecto que puede ser proyectado al desarrollo de programas para otras titulaciones que directa o indirectamente trabajen con mayores.

\section{Referencias}

Abellán, A., \& Esparza, C. (2011). Un perfil de las personas mayores en España, 2011. Indicadores demográficos básicos. Madrid: IMSERSO.

Abreu, M., \& Caldevilla, N. (2015). Attitudes toward aging in portuguese nursing students. Procedia-Social and Behavioral Sciences, 171, 961-967. doi: 10.1016/j. sbspro.2015.01.215

Aristizábal-Vallejo, N., Morales, A., Salas, B., Torres A. (2009). Estereotipos negativos hacia los adultos mayores en estudiantes universitarios. Cuadernos Hispanoamericanos de Psicología, 9(1), 35-44. Recuperado de https://m.uelbosque.edu.co/sites/default/files/publicaciones/revistas/ cuadernos_hispanoamericanos_psicologia/volumen9_nu mero1/articulo_3.pdf

Ayalon, L. (2013). Feelings towards older vs. younger adults: results from the European social survey. Educational Gerontology, 39(12), 888-901. doi: 10.1080/03601277.2013.767620

Barón, R., \& Byrne, D. (2005). Psicología social. Marid: Prentice Hall.

Blanca, M., Sánchez, C., \& Trianes, M. (2005). Cuestionario de evaluación de estereotipos negativos hacia la vejez. Revista Multidisciplinar de Gerontología, 15(4), 212-221.

Bousfield, C., \& Hutchison, P. (2010). Contact, anxiety, and young people's attitudes and behavioral intentions towards the elderly. Educational Gerontology, 36(6), 451-466. doi: 10.1080/03601270903324362

Bustillos, A., \& Fernández-Ballesteros, R. (2013). Attributions of competence mediate the behaviors of caregivers and older adults. GeroPsych: The Journal of Gerontopsychology and Geriatric Psychiatry, 26(4), 211-217. doi: 10.1024/1662-9647/a000089

Butler, R. N. (1980). Ageism: A foreword. Journal of Social Issues, 36(2), 8-11. doi: 10.1111/j.1540-4560.1980. tb02018.x

Campos, M., \& Salgado, E. (2013). Percepción sobre la tercera edad en estudiantes de primer nivel de la Facultad de Psicología de ULACIT y su relación con el desarrollo de competencias profesionales para el trabajo con adultos mayores. Revista Rhombus, 10(8), 1-30. 
Carver, C. S., \& Connor-Smith, J. (2010). Personality and coping. Annual Review of Psychology, 61, 679-704. doi: 10.1146/annurev.psych.093008.100352

Casado, J. M. R., Bustillos, A., Vaquero, A. I. G., Casal, C. H., \& Fernández-Ballesteros, R. (2016). ¿Se discrimina a los mayores en función de su edad? Visión del profesional. Revista Española de Geriatría y Gerontología, 51(5), 270275. doi: 10.1016/j.regg.2016.03.005

Chalabaev, A., Emile, M., Corrion, K., Stephan, Y., ClémentGuillotin, C., Pradier, C., \& d'Arripe-Longueville, F. (2013). Development and validation of the aging stereotypes and exercise scale. Journal of aging and physical activity, 21(3), 319-334. doi: 10.1123/japa.21.3.319

Chamorro, J. S. (2014). Imágenes sobre la vejez en jóvenes estudiantes universitarios según área del conocimiento: Un estudio exploratorio. Acciones e Investigaciones Sociales, 34, 105-124. doi: 10.26754/ojs_ais/ais.201434913

Charles, S. T., \& Carstensen, L. L. (2009). Socioemotional selectivity theory. En H. Reis \& S. Sprecher (Eds.), Encyclopedia of Human Relationships (pp. 1578-1581). California, SAGE Publications, Inc.

Chrisler, J. C., Barney, A., \& Palatino, B. (2017). Ageism can be hazardous to women's health: ageism, sexism, and stereotypes of oder women in the healthcare system. Journal of Social Issues, 72(1), 86-104. doi:10.1111/josi.12157

Duran-Badillo, T., Miranda-Posadas, C., Cruz-Barrera, L. G., Martínez-Aguilar, M., Gutiérrez-Sánchez, G., \& AguilarHernández, R. M. (2016). Estereotipos negativos sobre la vejez en estudiantes universitarios de enfermería. Revista de Enfermería del Instituto Mexicano del Seguro Social, 24(3), 205-209.

Fernández, E., Monardes, H., Díaz, C., Fuentes, F., \& Padilla, P. (2017). "Ageism" as social problem in geriatrics: Regarding the measurement of ageism in dentistry students. Revista Española de Geriatría y Gerontología, 52(2), 109. doi: 10.1016/j.regg.2016.08.003

Fernández-Ballesteros, R., Olmos, R., Santacreu, M., Bustillos, A., \& Molina, M. A. (2017). The role of perceived discrimination on active aging. Archives of Gerontology and Geriatrics, 71, 14-20. doi: 10.1016/j.archger.2017.02.004

Fuentes, C. L. C. (2014). Análisis de la relación entre las actitudes hacia la vejez y el envejecimiento y los índices de bienestar en una muestra de personas mayores. Revista Española de Geriatría y Gerontología, 49(3), 108-114. doi: 10.1016/j.regg.2013.06.001

Gázquez, J. J., Pérez-Fuentes, M. C., Fernández, M., González, L., Ruiz, I., \& Díaz, A. (2009). Old-age stereotypes related to the gerontology education: an intergenerational study. European Journal of Education and Psychology, 2(3), 263273. doi: $10.30552 /$ ejep.v2i3.27

Kornadt, A., \& Rothermund, K. (2015). Views on aging: Domain-specific approaches and implications for developmental regulation. Annual Review of Gerontology and Geriatrics, 35(1), 121-144. doi: 10.1891/0198-8794.35.121
Kydd, A., \& Fleming, A. (2015). Ageism and age discrimination in health care: Fact or fiction? A narrative review of the literature. Maturitas, 81(4), 432-438. doi: 10.1016/j. maturitas.2015.05.002

León, S., Correa-Beltrán, G., \& Giacaman, R. A. (2015). Negative ageing stereotypes in students and faculty members from three health science schools. Gerodontology, 32, 141-148. doi: 10.1111/ger.12065

Levy, B. R. (2003). Mind matters: Cognitive and physical effects of aging self-stereotypes. The Journals of Gerontology Series B: Psychological Sciences and Social Sciences, 58(4), 203-211. doi: 10.1093/geronb/58.4.P203

Losada-Baltar, A. (2004). Edadismo: consecuencias de los estereotipos, del prejuicio y la discriminación en la atención a las personas mayores. Algunas pautas para la intervención. Madrid: Portal Mayores, Informes Portal Mayores.

Márquez-González, M. (2010). Nuevas herramientas para la intervención psicológica con personas mayores: la tercera generación de terapias conductuales. Revista Española de Geriatría y Gerontología, 45(5), 247-249. doi: 10.1016/j. regg.2010.04.005

Mayordomo, T., Sales, A., Satorres, E., \& Meléndez, J. C. (2016). Bienestar psicológico en función de la etapa de vida, el sexo y su interacción. Pensamiento Psicológico, 14(2), 101-112. doi: 10.11144/Javerianacali.PPSI14-2.bpfe

Mehrotra, C., Townsend, A., \& Berkman, B. (2009). Enhancing research capacity in gerontologicalsocial work. Educational Gerontology, 35(2), 146-163. doi: 10.1080/03601270802421376

Meléndez, J. C., Tomás, J. M., \& Navarro, E. (2007). Análisis de las redes sociales en la vejez a través de la entrevista Manheim. Salud Pública de México, 49, 408-414. doi: 10.1590/S0036-36342007000600007

Montorio, I., Márquez, M., Losada, A., \& Fernández, M. (2003). Barreras para el acceso a los servicios de intervención psicosocial por parte de las personas mayores. Psychosocial Intervention, 12(3), 301-324.

Naciones Unidas. (2017). World Population Prospects: The 2017 Revision, Key Findings and Advance Tables. Working Paper No. ESA/P/WP/248. Department of Economic and Social Affairs, Population Division, United Nations.

Nelson, T. D. (2005). Ageism: Prejudice against our feared future self. Journal of Social Issues, 61(2), 207-221. doi: 10.1111/j.1540-4560.2005.00402.x

Rivera, L. M., \& Paredez, S. M. (2014). Stereotypes can "get under the skin": Testing a self-stereotyping and psychological resource model of overweight and obesity. Journal of Social Issues, 70(2), 226-240. doi: 10.1111/josi.12057

Robinson, S., Briggs, R., \& O’Neill, D. (2012). Cognitive aging, geriatric text books, and unintentional ageism. Journal of the American Geriatric Society, 60(11), 2183-2185. doi: 10.1111/j.1532-5415.2012.04217.x 
Rubio, M. J., \& Berlanga, V. (2012). Cómo aplicar las pruebas paramétricas bivariadas $t$ de Student y ANOVA en SPSS. REIRE. Revista d'Innovació i Recerca en Educació, 5(2), 83-100. doi: 10.1344/reire2012.5.2527

Sandoval, W. D., Galindo, M. C. E., Figueroa, D. G. I., Pulido, M. S. V., \& Ruvalcaba, N. M. M. (2016). Ideas sobre la vejez en estudiantes de Ciencias de la Salud del Centro Universitario de Tonalá. Investigación y Práctica en Psicología del Desarrollo, 1(1), 237-244.

Sanhueza, J. (2014). Estereotipos sociales sobre la vejez en estudiantes mayores: un estudio de caso. Revista Internacional de Educación para la Justicia Social. RIEJS [Internet], 3(1), 217-229.

Sanz, R., Mula, J. M., \& Moril, R. (2011). La relación abuelosnietos-escuela: una excusa o una necesidad. En Conferencia llevada a cabo en el XII Congreso Internacional de Teoría de la Educación. Universitat de Barcelona, Barcelona, España.

Sarabia, C. M., \& Castanedo, C. (2015). Modificación de estereotipos negativos en la vejez en estudiantes de enfermería. Gerokomos, 26(1), 10-12. doi: 10.4321/ S1134-928X2015000100003

Teixeira, M. C., Settembre, F. M., \& Leal, S. B. (2007). A survey of women's social representations of aging and rejuvenation. The Spanish Journal of Psychology, 10(1), 104-114. doi: $10.1017 / \mathrm{S} 1138741600006363$

Zambrini, D. A., Moraru, M., Hanna, M., Kalache, A., \& Nuñez, J. F. (2008). Attitudes toward the elderly among students of health care related studies at the University of Salamanca, Spain. Journal of Continuing Education in the Health Professions, 28(2), 86-90. doi: 10.1002/chp.162 